\title{
Molecular and cellular mechanisms of neutral lipid accumulation in diatom following nitrogen deprivation
}

\author{
Zhi-Kai Yang ${ }^{1}$, Ying-Fang Niu', Yu-Han Ma', Jiao Xue ${ }^{1}$, Meng-Han Zhang ${ }^{1}$, Wei-Dong Yang ${ }^{1}$, Jie-Sheng Liu', \\ Song-Hui Lu', Yuanfang Guan ${ }^{2^{*}}$ and Hong-Ye Li ${ }^{1 *}$
}

\begin{abstract}
Background: Nitrogen limitation can induce neutral lipid accumulation in microalgae, as well as inhibiting their growth. Therefore, to obtain cultures with both high biomass and high lipid contents, and explore the lipid accumulation mechanisms, we implemented nitrogen deprivation in a model diatom Phaeodacty/um tricornutum at late exponential phase.

Results: Neutral lipid contents per cell subsequently increased 2.4-fold, both the number and total volume of oil bodies increased markedly, and cell density rose slightly. Transcriptional profile analyzed by RNA-Seq showed that expression levels of 1213 genes (including key carbon fixation, TCA cycle, glycerolipid metabolism and nitrogen assimilation genes) increased, with a false discovery rate cut-off of 0.001 , under $\mathrm{N}$ deprivation. However, most light harvesting complex genes were down-regulated, extensive degradation of chloroplast membranes was observed under an electron microscope, and photosynthetic efficiency declined. Further identification of lipid classes showed that levels of MGDG and DGDG, the main lipid components of chloroplast membranes, dramatically decreased and triacylglycerol (TAG) levels significantly rose, indicating that intracellular membrane remodeling substantially contributed to the neutral lipid accumulation.
\end{abstract}

Conclusions: Our findings shed light on the molecular mechanisms of neutral lipid accumulation and the key genes involved in lipid metabolism in diatoms. They also provide indications of possible strategies for improving microalgal biodiesel production.

Keywords: Microalga, Nitrogen deprivation, Lipid, Membrane remodeling, Transcriptomics

\section{Background}

Concerns about global climate change and rises in prices of fossil fuels have prompted intense interest in the ability of microalgae to produce lipids that can be easily converted to biodiesel. Diatoms account for up to $40 \%$ of primary productivity in marine ecosystems [1] and some species are known to accumulate neutral lipids. Moreover, diatoms are metabolically versatile since they can synthesize and accumulate wide ranges of valuable compounds, such as polyunsaturated fatty acids (PUFAs), extracellular polymeric substances and cell coatings [2]. Hence, they have attracted

\footnotetext{
* Correspondence: gyuanfan@umich.edu; thyli@jnu.edu.cn

${ }^{2}$ Department of Computational Medicine and Bioinformatics, University of Michigan, Ann Arbor, Michigan, 48109, United States of America

'Key Laboratory of Eutrophication and Prevention of Red Tide of Guangdong Higher Education Institute, College of Life Science, Jinan University, Guangzhou, 510632, China
}

both biological and medical attention. Recently, the marine diatom Phaeodactylum tricornutum has emerged as a potential microalgal energy source. It grows rapidly, has a short life cycle, and accumulates TAGs in late exponential phase; storage lipids constitute about $20-30 \%$ of its dry cell weight under standard culture conditions [3]. Furthermore, it is a model diatom species and its genome has been fully sequenced and available at the JGI (http://genome.jgi-psf. org/Phatr2/Phatr2.home.html) [4].

The accumulation of neutral lipids and changes in lipid profiles under nutrient starvation have been monitored in $P$. tricornutum and another model diatom, Thalassiosira pseudonana [5-7]. Nitrogen is a major constituent of proteins and nucleic acids. Accordingly, pigment and protein losses have been observed in Antarctic sea ice diatoms under nitrogen limitation [8], and while

\section{Biomed Central}


N-starved $P$. tricornutum cells can accumulate high levels of lipids, their division reportedly ceases and cell density increases only marginally $[1,9,10]$. Furthermore, changes in fatty acid profiles of the green alga Chlamydomonas reinhardtii under $\mathrm{N}$ deprivation have been reported recently [11], and associated shifts in fluxes through metabolic pathways have been inferred from changes in transcript abundance in C. reinhardtii [12]. However, the biochemical and molecular mechanisms involved in diatom responses to losses of nitrogen availability are still unclear. Therefore, in the study reported here P. tricornutum was cultured under standard laboratory conditions then subjected to nitrogen deprivation in the late exponential phase to achieve both high neutral lipid contents and high cell biomass. Then, to assess the holistic effects of $\mathrm{N}$ deprivation and identify the mechanism involved in the diatoms, we examined changes in their levels of neutral lipids, oil bodies, fatty acid profiles, chloroplast structure, photosynthetic parameters, gene expression patterns and inferred shifts in fluxes through key metabolic pathways.

\section{Results and discussion}

Effects of $\mathrm{N}$ deprivation in late exponential growth phase on growth and neutral lipid accumulation in $P$. tricornutum To monitor their growth, the $P$. tricornutum cultures were sampled at the same time (14:00) daily and cells in the samples were counted using a hemacytometer. As shown in Figure 1, the cultures grown solely under standard conditions showed typical growth curves. Switching to fresh medium (controls) in late exponential phase stimulated growth, and switching to $\mathrm{N}$-free medium ( $\mathrm{N}$ deprivation, $-\mathrm{N}$ )

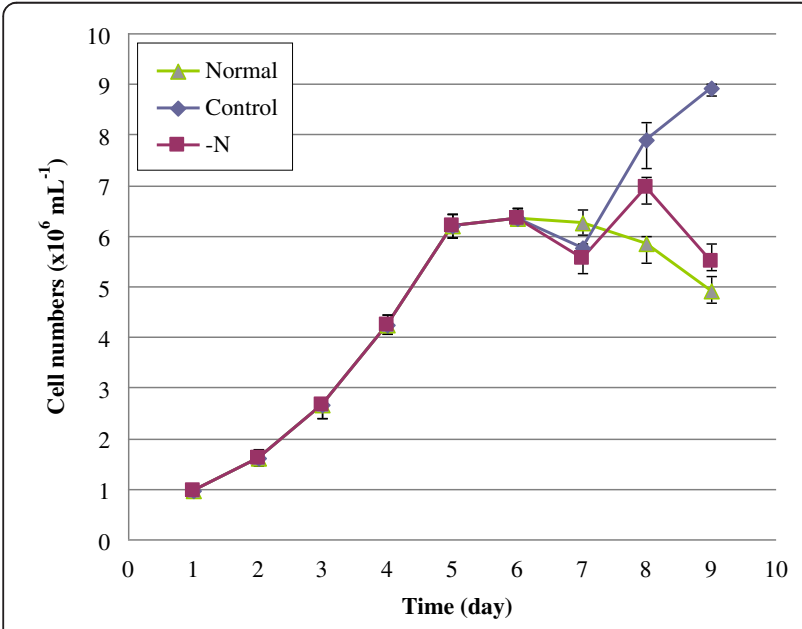

Figure 1 Growth of $P$. tricornutum under standard and $\mathrm{N}$-free conditions. Normal, cells grown under standard conditions, i.e. continuous culture in f/2-Si medium; Control, cells cultured in f/2-Si medium, with a transfer to fresh medium 5 days after subculture; $-\mathrm{N}$, cells cultured in $\mathrm{f} / 2$-Si medium, with a transfer to fresh $\mathrm{N}$-free medium 5 days after subculture. induced an intermediate response; slightly lower growth than in the controls, but higher than that of the standard continuous cultures. These findings indicate that $\mathrm{N}$ deprivation in late exponential phase can stimulate further increases in cell biomass, and intracellular $\mathrm{N}$ reserves can be used by diatom cells to fuel growth even if the extracellular $\mathrm{N}$ concentration is low. It should be noted that the cells showed typical growth characteristics in the absence of silicon, in accordance with findings by De Martino et al. [13]. Thus, silicon was omitted from the culture medium used in further experiments, to prevent the formation of frustules and thus facilitate cell breakage.

The lipid contents of the cultures were assessed, rapidly and conveniently, using Nile Red and a fluorimetric spectrophotometer with a $480 \mathrm{~nm}$ excitation wavelength. The optimal emission and staining time were empirically found to be $592 \mathrm{~nm}$ and $20 \mathrm{~min}$, respectively, similar to previously optima for neutral lipid determination: $480 \mathrm{~nm}$ and 580-610 nm emission and excitation wavelengths, respectively, with 10-30 min staining time [14]. The fluorescence intensity per cell was significantly $(10 \%)$ higher than that of the controls after one day of $\mathrm{N}$ deprivation, and 2.4-fold higher after two days (Figure 2), which was basically in line with the lipid contents of $24.13 \%$ (control) and $53.33 \%(-\mathrm{N})$ obtained after $-\mathrm{N}$ for 2 days by the gravimetric determination (Table 1). These results confirmed that $\mathrm{N}$-deprivation induces substantial neutral lipid accumulation and that $P$. tricornutum is a promising biodiesel resource. However, the growth rate of the $\mathrm{N}$-deprived cultures was slightly lower than control rates following the medium changes, although higher than that of the cultures kept in the same medium throughout the experiment. The findings indicate that a two-step culture strategy with $\mathrm{N}$ deprivation could deliver much higher neutral lipid yields than strategies based simply on continuous $\mathrm{N}$ deficiency [15].

\section{Changes in fatty acid composition under $\mathbf{N}$ deprivation}

As shown in Table 1, substantial differences in fatty acid composition between N-deprived and control cells were detected by GC-MS analysis: proportions of saturated fatty acids (SFA) and monounsaturated fatty acids (MUFA) were $45 \%$ and $61 \%$ higher in the former, respectively, while the proportion of polyunsaturated fatty acids (PUFA) was $22 \%$ lower. The proportions of other fatty acids also markedly declined in the N-deprived cultures. The total unsaturated lipid content decreased by $16 \%$ under $\mathrm{N}$ deprivation, and proportions of the long-chain fatty acids C20:5 and C22:6 were $43 \%$ and $40 \%$ lower, respectively. The changes in fatty acid composition were largely due to dramatic increases in palmitic and palmitoleic acids, with accompanying losses of eicosapentaenoic acid, which is generally one of the most abundant fatty acids in P. tricornutum [16]. 


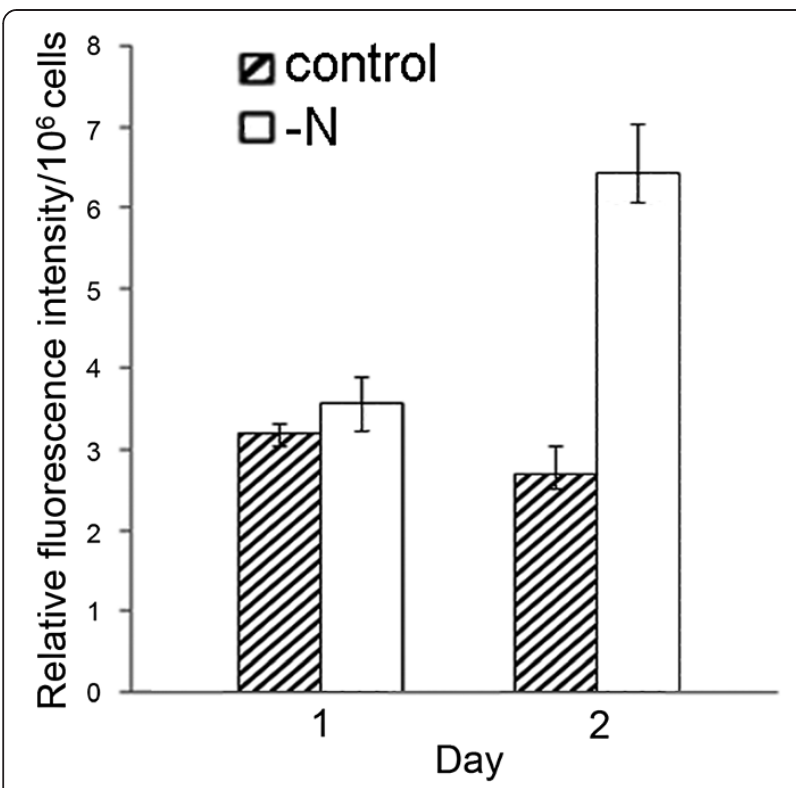

Figure 2 Relative fluorescence intensity of diatom cells under $\mathbf{N}$ deprivation. Triplicate samples of diatom cells were stained with Nile Red after 1 and 2 days of $\mathrm{N}$ deprivation, their fluorescence was measured and their relative fluorescence intensity was calculated by subtracting the autofluorescence of non-stained microalgae and Nile red. The left and right columns in each day reprensent the control and $-\mathrm{N}$, respectively.

\section{Observation of oil bodies by confocal microscopy}

The size and number of oil bodies in sampled cells were observed every day during the culture cycle using a laser scanning confocal microscope. Substantially more oil bodies were observed in the $\mathrm{N}$-deprived cells than in controls, but their sizes were similar (Figure 3), with diameters ranging from 0.2 to $2.0 \mu \mathrm{m}$. Thus, the total volume of oil bodies was clearly higher in the $\mathrm{N}$-deprived cultures than in the controls, in accordance with the neutral lipid increase, as shown in Figure 2.

\section{High-throughput analysis of differential gene expression}

To characterize the effects of $\mathrm{N}$ deprivation further, we examined differences in gene expression before and after $\mathrm{N}$-deprivation, using RNA-Seq. The complete dataset is listed in Additional file 1, and also available at http:// guanlab.ccmb.med.umich.edu/data/Yang_N_deprivation/, but briefly 1213 genes were found to be up-regulated, with an FDR cutoff of $0.1 \%$, and 4527 genes were downregulated, indicating that the deprivation induced wide reprogramming of regulation. Distribution of the number of reads across different mRNA sequence size indicates a significant correlation (Additional file 2: Figure S1). To validate the findings we applied quantitative RT-PCR (qPCR) to a randomly selected set of genes, including $\beta$-actin as a housekeeping marker, to test the differential expression. The RNA-Seq measurements are highly consistent with the quantitative PCR results, confirming their robustness.
Table 1 Fatty acid composition of $P$. tricornutum following $\mathbf{N}$ deprivation

\begin{tabular}{|c|c|c|c|c|}
\hline \multirow[t]{2}{*}{ Fatty acids } & \multicolumn{2}{|c|}{ Control } & \multicolumn{2}{|l|}{$-\mathrm{N}$} \\
\hline & Total FA\% & DW\% & Total FA\% & DW\% \\
\hline C14:0 (myristic) & 5.31 & 0.22 & 6.27 & 0.12 \\
\hline C16:0 (palmitic) & 15.89 & 0.66 & 25.87 & 0.49 \\
\hline C18:0 (stearic) & 2.67 & 0.11 & 2.37 & 0.04 \\
\hline Sum SFA & 23.87 & 0.99 & 34.51 & 0.65 \\
\hline C16:1 (palmitoleic) & 23.91 & 0.99 & 42.55 & 0.80 \\
\hline C18:1 (oleic) & 2.45 & 0.10 & NT & ND \\
\hline Sum MUFA & 26.36 & 1.09 & 42.55 & 0.80 \\
\hline C18:2 (linoleic) & NT & NT & 5.22 & 0.10 \\
\hline C18:3 (linolenic) & 2.69 & 0.11 & 1.25 & 0.02 \\
\hline C20:5 (eicosapentaenoic) & 20.33 & 0.84 & 11.67 & 0.22 \\
\hline C22:6 (docosahexaenoic) & 1.07 & 0.04 & 0.64 & 0.01 \\
\hline Sum PUFA & 24.09 & 1.00 & 18.78 & 0.35 \\
\hline other FA & 10.88 & 0.45 & 2.71 & 0.05 \\
\hline Total FA identified & 85.20 & 3.53 & 98.55 & 1.85 \\
\hline \multicolumn{5}{|l|}{ Unsaturation ratios: } \\
\hline C 16 unsat./C16:0 & 1.50 & 0.06 & 1.64 & 0.03 \\
\hline C18 unsat./C18.0 & 1.93 & 0.08 & 2.73 & 0.05 \\
\hline Total unsat./total sat. & 2.11 & 0.09 & 1.78 & 0.03 \\
\hline Lipid content (DW) & & 24.13 & & 53.33 \\
\hline
\end{tabular}

Abbreviations: FA, Fatty acid; DW, Dry weight; SFA, Saturated fatty acid; MUFA, Monounsaturated fatty acid; PUFA, Polyunsaturated fatty acid; $N D$, not detected.

For example, Phatrdraft_27877, encoding an ammonium transporter protein, was up-regulated $5.46\left(\log _{2}\right)$-fold and 116-fold under N-deprivation, according to the RNA-Seq and GPCR analyses, respectively (Table 2).

\section{General transcriptional changes under $\mathbf{N}$ deprivation}

To elucidate the molecular basis of the observed accumulation of fatty acids under $\mathrm{N}$ deprivation, genes encoding all known enzymes in the $P$. tricornutum genome were mapped in KEGG pathways, together with $\log _{2}$-fold differences in RNA expression between the control and $\mathrm{N}$-deprived cultures. Global changes in major categories of genes involved in various pathways, reflecting general transcriptional responses to $\mathrm{N}$ deprivation, are depicted in Figure 4 and listed in Additional file 3: Table S1a. Genes encoding photosynthesis, gluconeogenesis, glyoxylate cycle, chrysolaminarin synthesis and sucrose metabolism enzymes were, on average, significantly down-regulated under $\mathrm{N}$ deprivation, while genes involved in nitrogen fixation, carbon fixation, glycolysis and the TCA cycle were generally up-regulated.

The primary effect of $\mathrm{N}$ deprivation is the reduced availability of nitrogen, an essential nutrient for de novo synthesis of amino acids, nucleic acids and various other cellular constituents. Unsurprisingly, therefore, it strongly affected transcript levels of genes involved in 

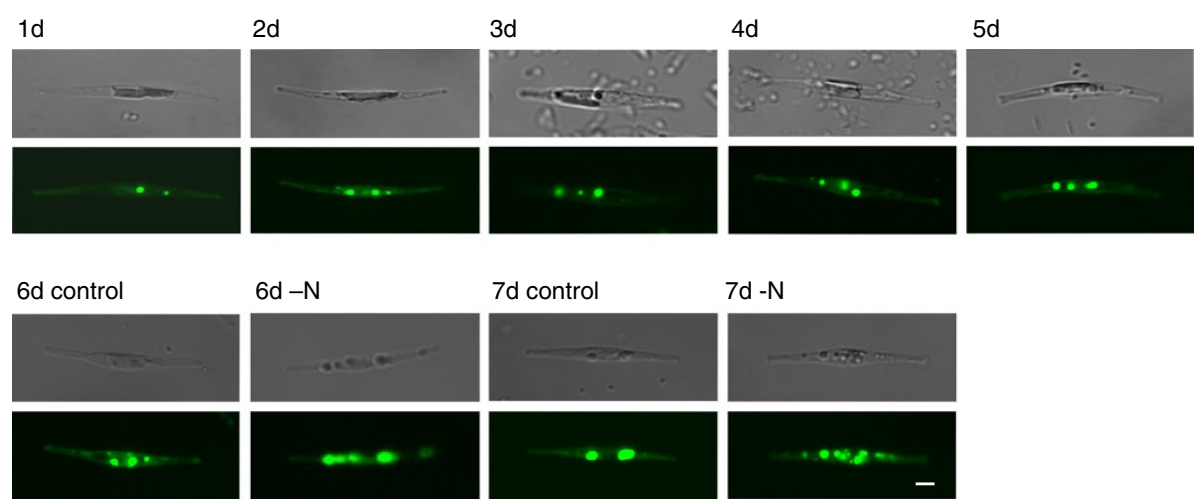

Figure 3 Representative confocal microscope images of the diatoms, showing oil bodies. Cells sampled during a 7-day culture cycle were stained with Nile Red and photographed under the confocal microscope daily. $-\mathrm{N}$, cells subjected to the $\mathrm{N}$ deprivation treatment five days after subculture; Control, cells grown in f/2-Si medium and switched to fresh medium five days after subculture. Bar $=2 \mu \mathrm{m}$.

nitrogen assimilation and metabolism (Additional file 3: Table S1b). Notably, marked increases were observed in transcripts encoding five of at least six ammonium transporters present in the diatom, which transport ammonium ions across the cell membrane and are known to be activated by $\mathrm{N}$ deprivation [17]. The rise in the level of one of them was also confirmed in our qPCR test (Phatrdraft_27877, 45-fold increase). In addition, there was a 7-fold increase in transcripts of glutamine synthase (GS, Phatrdraft_22357, EC 6.3.1.2), a key enzyme in ammonia assimilation in both plants and Gram-negative microorganisms. GS plays an important role in the efficient use of nitrogen sources and nitrogen metabolism. Other critical nitrogen metabolism enzymes, such as nitrate reductase (Phatrdraft_54983, EC1.7.7.2), a molybdoenzyme that reduces nitrate to nitrite, and ferredoxin-nitrite reductase (Phatrdraft_12902, EC1.7.7.1), which catalyzes conversion of nitrite to ammonia, also showed significant increases (4-fold and 33-fold, respectively) in transcript abundance. These observations indicate that $\mathrm{N}$ deprivation induces homeostatic responses, including activation of the glutamine synthesis pathway and increases in the cells' capacity to utilize trace amounts of nitrogen resources, and also the possible redistribution of intracellular nitrogen such as increased amino acid catabolism in accordance with the study in T. pseudonana [6], ultimately yielding acetyl-CoA or succinyl-CoA as the TCA cycle intermediates.

\section{Transcripts of photosynthesis-related genes decreased}

As shown in Additional file 3: Table S1c, levels of most transcripts encoding proteins associated with photosynthesis decreased under $\mathrm{N}$ deprivation, implicating that photosynthesis could be inhibited or at a standstill. Notably, only one encoding a light harvesting complex (LHC) component was up-regulated (Phatrdraft_16481, fucoxanthin chlorophyll a/c protein; up-regulated 10 -fold), while most of the others were down-regulated more than 10-fold. As previously demonstrated in $C$. reinhardtii, reductions in the abundance of light-harvesting complexes may reduce photosynthetic rates [18], in accordance with theoretical expectations, unless reductions in transcript levels are balanced by changes at other levels. Transcript levels of ferredoxin-NADP ${ }^{+}$reductase (Phatrdraft_43018), which catalyzes the last electron transfer, from photosystem I

Table 2 qPCR of differentially expressed genes following $\mathbf{N}$ deprivation

\begin{tabular}{|c|c|c|c|c|c|c|}
\hline \multicolumn{4}{|c|}{ qPCR } & \multicolumn{3}{|c|}{ RNA-Seq } \\
\hline Locus tag & Forward $\left(5^{\prime}-3^{\prime}\right)$ & Reverse $\left(5^{\prime}-3^{\prime}\right)$ & $\mathrm{FC}(\mathrm{N} / \mathrm{Ctr})$ & $\mathrm{FC} \log _{2}(\mathrm{~N} / \mathrm{Ctr})$ & Z-test & FDR \\
\hline 54257 & agaatcggtgatgcctgttc & atgccgctgctttagtgaat & 103.3 & 5.80 & 0 & 0 \\
\hline 45012 & acgattcggacgaagatcag & ccatgcaacaatcgtagtgg & 26.0 & 5.56 & $6.71 \mathrm{E}-14$ & $1.99 \mathrm{E}-13$ \\
\hline 27877 & acaccaccgacaagaccttc & tccagacagagcgtacaacg & 116.6 & 5.46 & 0 & 0 \\
\hline 54465 & gagcacttcgttctccaagg & gtccagaaagccacagcttc & -17.1 & -8.98 & 0 & 0 \\
\hline 13154 & caggaactcgcgaagttagg & gcaagaatggaacccactgt & 3.35 & 7.42 & $2.46 \mathrm{E}-5$ & $4.37 E-5$ \\
\hline 45852 & aaggccacaatctcatggac & cttttgacggatggcaactt & 23.6 & 7.41 & 7.47E-9 & $1.63 \mathrm{E}-8$ \\
\hline 51183 & ctttacaacgecctgatcgt & ttgctgtcgtggaaagactg & -15.7 & -8.65 & 0 & 0 \\
\hline 9794 & gcatattggaggctttggaa & tctgcatcatcatcccgata & 1.35 & -0.58 & $7.29 \mathrm{E}-3$ & 0.010 \\
\hline
\end{tabular}

Each value corresponds to the mean of three biological sample performed in duplicates; $\beta$ - actin is used as house-keeping gene in real-time quantitative PCR; significant changes are indicated by a $p$ value $<0.05$ (Z-test). 


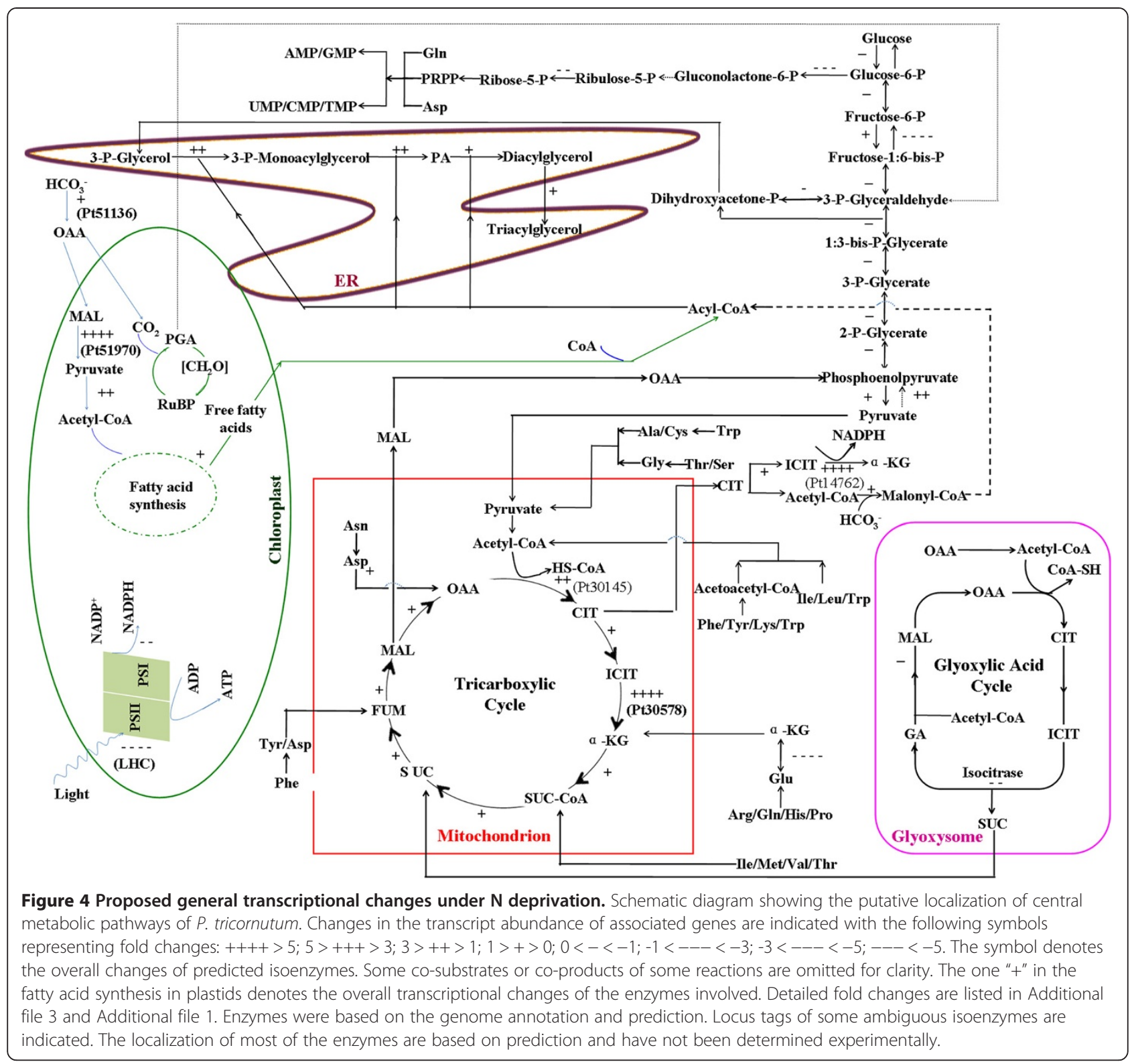

to NADPH during photosynthesis [19], also decreased (4.6-fold).

The reductions in mRNA levels of genes encoding photosynthetic proteins under $\mathrm{N}$ deprivation were consistent with expectations since various stresses can inhibit photosynthesis, and upregulated light harvesting can result in excess production of highly toxic reactive oxygen species (ROS) [20]. To examine whether the reduced expression of photosynthetic genes was reflected in physiological changes, we also measured and calculated chlorophyll fluorescence parameters of the cultures, including Fo, Fm, Fv/Fm and Fv/Fo. As shown in Table 3, all of these parameters significantly decreased under $\mathrm{N}$ deprivation, indicating that the stress impaired PSII reaction centers, reduced the chlorophyll fluorescence yield, and decreased both the primary light energy conversion efficiency and potential photochemical activity of the PSII reaction centers [21]. The inhibition of genes associated with photosynthesis by $\mathrm{N}$ deprivation observed in this study is consistent with reported responses of $P$. tricornutum [7] and green alga Chlamydomonas [12,22], and can be considered a generic response to nutrient deficiencies. A practical implication of the findings for industrial biodiesel production is that light may not be required for neutral lipid accumulation under $\mathrm{N}$ deprivation. However, a previous report indicates that polysaccharides are produced at high rates during light periods and consumed in the dark, while the total lipid contribution to dry weight is positively associated with light intensity in $\mathrm{N}$-deficient $P$. tricornutum [9]. Thus, the effects of light on lipid accumulation in 
diatoms, and its interactive effects with other environmental factors, clearly require further characterization.

\section{Increased carbon assimilation and carbon fluxes towards TAG accumulation}

Two genes encoding putative isoforms of phosphoenolpyruvate carboxylase (PEPC, EC4.1.1.31), Phatrdraft_27976 and Phatrdraft_51136, are present in the P. tricornutum draft genome and both were up-regulated moderately under $\mathrm{N}$ deprivation, 1.5-fold and 2-fold, respectively (Additional file 3: Table S1d). The two PEPCs were predicted to be localized in the chloroplast (Phatrdraft_27976; mito: 7.0, chlo: 7.0; Phatrdraft_51136, chlo: 9.0, cyto: 5.0, by WoLF PSORT) implicating the presence of C4 photosynthetic pathway. Two green microalgae, C. reinhardtii and Selenastrum minutum, also reportedly possess at least two distinct PEPC isoforms, which differ significantly from their plant and prokaryotic counterparts [23]. PEPC catalyzes the irreversible $\beta$-carboxylation of phosphoenolpyruvate (PEP) in the presence of $\mathrm{HCO}_{3}$ - and $\mathrm{Me}^{2+}$ to yield inorganic phosphate and oxaloacetate (OAA). Thus, it is intimately involved in $\mathrm{C}_{4}$-dicarboxylic acid metabolism in these organisms. It also plays an anaplerotic role in most non-photosynthetic organs of plants, $\mathrm{C}_{3}$ leaves and microalgae (including diatoms), providing OAA and/or malate to replenish citric acid cycle intermediates consumed in other primary metabolic pathways, notably ammonia assimilation [24]. PEPC is considered to be a key enzyme for carbon fixation in diatoms $[18,24]$. In fact, the moderate up-regulation of the two PEPCs here was basically in accord with the other reports that $P$. tricornutum PEPC transcripts showed no significant increase under low $\mathrm{CO}_{2}$ conditions $[7,25]$. Although the carbon fixation pathway in P. tricornutum has not been fully elucidated, the deduced pathway in some diatoms $[18,25-27]$ and increases in transcript levels of both PEPCs observed here suggest that it can take up inorganic carbon and possesses a $\mathrm{CO}_{2}$ concentration mechanism (CCM) (Additional file 4: Figure S2).

In addition, transcript levels of a predicted malic enzyme (NADP ${ }^{+}$-dependent ME; EC1.1.1.40, Phatrdraft_51970), a component of the putative carbon fixation pathway, with a predicted chloroplast localization (chlo: 8.0, cyto: 6.0, by WoLF PSORT), dramatically increased, over 7-fold (Additional file 4: Figure S2; Additional file 3: Table S1d). ME catalyzes a rate-limiting step for fatty acid biosynthesis; the irreversible decarboxylation of malate to pyruvate with the formation of $\mathrm{NADPH}$ from $\mathrm{NADP}^{+}$, hence providing essential reducing power for fatty acid biosynthesis via fatty acid synthase. Another previously reported putative decarboxylating enzyme of the malic enzyme family encoded by Phatrdraft_56501 [27], was predicted to be mainly chloroplast location (chlo: 11.0 , mito: 2.0 , by WoLF PSORT). Its presence is also consistent with reports of a single-cell $\mathrm{C}_{4}$ pathway in marine diatoms, including $P$. tricornutum, involving OAA entering the chloroplast [25]. Wynn et al. have investigated the role of ME in filamentous fungi [28] and found that its overexpression increases NADPH production, thereby providing reducing power and cofactors for reactions catalyzed by enzymes involved in TAG synthesis such as ACCase, fatty acid synthase (FAS), ultimately leading to increases in TAG accumulation. Similarly, overexpression of two ME genes from Mucor circinelloides and Mortierella alpine in Mucor circinelloides reportedly increased ME activity 2- to 3-fold, and increased lipid contents from $12 \%$ to $30 \%$ of total biomass [29]. Therefore, the dramatic increase in ME transcription we observed under $\mathrm{N}$ deprivation could contribute substantially to the accumulation of neutral lipids in $P$. tricornutum. In contrast, levels of pyruvatephosphate dikinase (EC2.7.9.1, Phatrdraft_21988) transcripts decreased 7.5-fold. Pyruvate-phosphate dikinase is a key enzyme in the $C_{4}$ photosynthetic pathway [30], thus reduction in its expression could reduce photosynthesis rates. Under such circumstances, increases in PEPC expression or activities in P. tricornutum could optimize use of the available phosphoenolpyruvate for carbon fixation, while saving most pyruvate for acetyl-CoA formation. Further, decreases in pyruvate-phosphate dikinase activity may prevent possible activation of alternative gluconeogenic pathways, such as that reportedly observed in Rhizobium (Sinorhizobium) meliloti [30]. Taken together, the transcriptional changes in carbon assimilation under $\mathrm{N}$ deprivation may substantially increase carbon influxes, providing a rich source of substrate for fatty acid production. In fact, Fourier transform infrared spectrometer (FTIR) assay conducted according to Jiang et al. [31] showed a slight increase of carbohydrate under $\mathrm{N}$ deprivation (data not shown), which is basically in line with the previous findings in P. tricornutum [7] and T. pseudonana [31]. Furthermore, the 2-fold increase of

Table 3 Measurement of photosynthetic activity

\begin{tabular}{ccccr}
\hline Group & \multicolumn{4}{c}{ Chlorophyll fluorescence parameters } \\
\cline { 2 - 5 } & Fv/Fm & Fv/Fo & Fo & Fm \\
\hline Control & $0.604 \pm 0.003$ & $1.520 \pm 0.019$ & $323.75 \pm 2.50$ & $816.75 \pm 7.23$ \\
\hline$-\mathrm{N}$ & $0.550 \pm 0.0164^{* *}$ & $1.221 \pm 0.080^{* *}$ & $184.25 \pm 4.86^{* *}$ & $409.00 \pm 5.42^{* *}$ \\
\hline
\end{tabular}

Note: Chlorophyll fluorescence parameters were monitored $48 \mathrm{~h}$ post $\mathrm{N}$ deprivation $(-\mathrm{N})$. Date are shown as means $\overline{\times} \pm$ SD; ${ }^{*}$ and ${ }^{* *}$ denote significant difference $(p<0.05)$ and extremely significant difference $(p<0.01)$, respectively. 
$\mathrm{C}: \mathrm{N}$ ratio during $\mathrm{N}$ depletion according to Valenzuela et al. [7] together with the slight increase of carbohydrate implicate that the carbon is challenged into the production of storage lipid during $-\mathrm{N}$.

P. tricornutum appears to possess at least five isoforms of fructose-1,6-bisphosphatase (FBP, EC3.1.3.11), a key regulatory enzyme of carbon metabolism (particularly the Calvin cycle and gluconeogenesis), which catalyzes the conversion of fructose-1,6-biphosphate to fructose-6phosphate (Additional file 3: Table S1d). Transcript levels of genes encoding four of these isoforms, Phatrdraft_2793, Phatrdraft_9359, Phatrdraft_31994 and Phatrdraft_8744, declined under N deprivation, 45-, 17-, 3- and 3.5-fold, respectively, while those of the other (Phatrdraft_23247) slightly increased, by $50 \%$. Therefore, gluconeogenesis was markedly inhibited and carbon flux was re-directed towards TAG accumulation under $\mathrm{N}$ deprivation. In contrast, two genes encoding phosphofructokinase (EC2.7.1.11), which catalyzes the committed step in the glycolytic pathway (conversion of fructose-6-phosphate to fructose-1,6bisphosphate) were up-regulated: Phatrdraft_55126 and Phatrdraft_16844, by $36 \%$ and 50\%, respectively. The upregulation of glycolysis should theoretically direct carbon flux to the formation of pyruvate. Moreover, levels of transcripts encoding a precursor of the pyruvate dehydrogenase E1 component beta subunit (Phatrdraft_20183, EC1. 2.4.1) and the alpha subunit (Phatrdraft_55035) increased 3 -fold and slightly (by 15\%), respectively. These findings propose that conversion of pyruvate to acetyl-CoA may be activated under $\mathrm{N}$ deprivation, thereby providing substrate for the citric acid (TCA) cycle following the conversion of acetyl-CoA to citrate as well as building up precursors to ACCase for fatty acid biosynthesis. And we found that the predicted cytosolic isocitrate dehydrogenase (Phatrdraft_ 14762) increased 16-fold, which is involved in the conversion of citrate to $\alpha$-ketoglutarate. During this process, $\mathrm{NADP}^{+}$is reduced to NADPH which is a critical cofactor for many enzymatic reactions in lipid biosynthesis [32].

Diatoms do not possess starch-forming enzymes, but store fixed carbon as a complex, soluble carbohydrate called chrysolaminarin ( $\beta$-1, 3-glucan) in vacuoles. Transcript levels of phosphoglucomutase (Phatrdraft_50445), which catalyzes the rate-limiting step of carbohydrate synthesis, decreased 3.7-fold under $\mathrm{N}$ deprivation. This is consistent with both the observations of neutral lipid accumulation and recent reports that $\mathrm{N}$-deprived $C$. reinhardtii mutants with phosphoglucomutase deficiencies store neutral lipids in lipid bodies [33,34]. Furthermore, since chrysolaminarin and TAG syntheses share common 3-carbon photosynthate precursors, mutations or reductions in the expression of phosphoglucomutase could affect chrysolaminarin biosynthesis and redirect carbon fluxes to TAG accumulation. The decrease in carbohydrate contents under $\mathrm{N}$ deprivation is consistent with previous findings that high lipid contents in $P$. tricornutum are accompanied by low total carbohydrate contents [9].

Almost all of the genes involved in the TCA cyclewhich converts 2-oxoglutarate, coenzyme A and $\mathrm{NAD}(+)$ to succinyl-CoA, NADH and carbon dioxide-were activated under $\mathrm{N}$ deprivation (Additional file 4: Figure S2; Additional file 3: Table S1e). Levels of transcripts encoding two potentially rate-limiting enzymes of the TCA cyclecitrate synthase (Phatrdraft_30145, EC2.3.3.1) and isocitrate dehydrogenase (EC1.1.1.42, Phatrdraft_30578) increased 3- and 22-fold, respectively. In addition, those of a third TCA cycle enzyme, oxoglutarate dehydrogenase (Phatrdraft_29016, EC1.2.4.2), which converts 2-oxoglu tarate to succinyl-CoA [19], slightly increased under $\mathrm{N}$ deprivation. These findings suggest that TCA cycle genes were markedly up-regulated under $\mathrm{N}$ deprivation, thus increases in fluxes through the TCA cycle may compensate for the loss of assimilatory power for carbon fixation due to the down-regulation of photosynthesis.

The transcript abundance of isocitrate lyase (Phatrdraft 14401, EC4.1.3.1) and malate synthase (Phatrdraft_54478), two glyoxylate cycle enzymes identified in the $P$. tricornutum genome, decreased 5- and 2.5-fold, respectively. This implies that the metabolic flux through the glyoxylate cycle could be reduced under $\mathrm{N}$ deprivation, thereby further inhibiting overproduction of oxaloacetate and gluconeogenesis. Similarly, transcripts encoding phosphoenolpyruvate carboxykinase (Phatrdraft_55018), which catalyzes the committed step of gluconeogenesis, decreased by $20 \%$.

\section{Multiple sources for TAG accumulation and reductions in lipid catabolism}

Transcript levels of genes associated with TAG biosynthesis were up-regulated by N-deprivation (Additional file 3: Table S1f). Notably, mRNA levels of Phatrdraft_9794, encoding diacylglycerol acyltransferase (DGAT, EC2.3.1.20), increased by $30 \%$, according to both the RNA-Seq and qPCR analyses (Table 2). DGAT catalyzes the final committed step of TAG biosynthesis, thus the increase in its mRNA abundance under $\mathrm{N}$ deprivation may have increased TAG levels somewhat, but not as much as the observed increase in neutral lipid contents. Similarly, in C. reinhardtii there are two DGAT homologs, and transcripts encoding (DGTT2) are reportedly present at consistently low levels under all tested conditions, including $\mathrm{N}$ deprivation, while mRNA of the other (DGTT3) is present at low levels and only increases slightly under $\mathrm{N}$ deprivation [12]. The ability of increases in DGAT expression to raise TAG levels has been demonstrated by heterologous expression of $P$. tricornutum DGAT (PtDGAT1, Phatrdraft_9794) in a Saccharomyces cerevisiae neutral lipid-deficient quadruple mutant strain, which restored TAG and lipid body formation, and promoted 
incorporation of saturated fatty acids into TAGs [35]. Similarly, overexpression of Tropaeolum majus DGAT1 in Brassica napus and Arabidopsis thaliana resulted in 11-30\% net increases in seed oil content [36].

Comparing with the much more increase of neutral lipid levels in P. tricornutum under $\mathrm{N}$ deprivation (2.4-fold), the slight increase of DGAT transcripts could be less effective. We examined further potential sources of substrates for TAG accumulation, and found that mRNA levels of another enzyme, responsible for the last step of TAG biosynthesis, phospholipid:diacylglycerol acyltransferase (PDAT, Phatrdraft_8860, EC2.3.1.158), increased 2-fold. Interestingly, in addition to the DGAT-catalyzed pathway, another acyl-CoA independent pathway catalyzed by PDAT for TAG synthesis has been discovered in Arabidopsis, in which acyl is directly transferred from phosphatidylcholine (PC) to DAG and thus TAG is synthesized without use of CoA as an intermediate [37]. Phosphatidate phosphatase (PAP, EC3.1.3.4, Phatrdraft_40261) mRNA levels increased by $25 \%$, which were responsible for the intermediate DAG for PDAT-catalyzed TAG accumulation. These findings suggest that the PDAT-mediated pathway or intracellular membrane remodeling may have contributed to the observed TAG accumulation.

The transcript abundance of genes involved in fatty acid elongation decreased under $\mathrm{N}$ deprivation. Delta 6 elongase (Phatrdraft_20508) and long chain acyl-CoA elongase (Phatrdraft_34485) levels decreased by 16\% and $32 \%$, respectively, which is consistent with observed reductions in C20:5 and C22:6 fatty acids, as shown in Table 1. Responses of genes encoding fatty acid desaturases varied. Transcript abundance of two desaturases catalyzing PUFA formation (delta 12 fatty acid desaturase, Phatrdraft_25769, and delta 9 fatty acid desaturase, Phatrdraft_28797) decreased, by 2.6- and 4-fold, respectively. In contrast, the expression of delta 5 fatty acid desaturase (Phatrdraft_46830), involved in MUFA synthesis, increased dramatically (32fold). These results are in line with the higher MUFA and lower PUFA proportions of fatty acids under $\mathrm{N}$ deprivation (Table 1).

In accordance with the general increases in transcript levels of genes involved in TAG synthesis, levels of those involved in lipid catabolism generally decreased under $\mathrm{N}$ deprivation, those of acyl-CoA oxidase (Phatrdraft_19979) and 3-oxoacyl-CoA thiolase (ATO1) most dramatically; 11-fold and more than 3-fold, respectively.

Lipases are enzymes that de-esterify carboxyl esters, such as TAGs and phospholipids. More than 28 genes encoding putative lipases are present in the $P$. tricornutum genome (Additional file 3: Table S1g) and their responses to $\mathrm{N}$ deprivation varied markedly. Since TAG accumulated markedly under $\mathrm{N}$ deprivation, TAG lipases would be expected to be down-regulated. Among the putative lipase genes, eight (28\%) showed increased transcript abundance but mainly only slightly, and transcript levels of most of the others decreased more than 2-fold. For example: Phatrdraft_44231, which encodes a putative triacylglycerol lipase, decreased 5-fold; Phatrdraft_50397 decreased 4fold; while 43593 increased nearly 4-fold. These lipase genes may play important roles in membrane turnover, and their overall reduction in transcription may protect the TAG from degradation, thereby promoting remodeling and TAG accumulation under $\mathrm{N}$ deprivation.

\section{Effects of $\mathbf{N}$ deprivation on the subcellular ultrastructure}

Sections of cells were observed by transmission electron microscopy to assess the impact of $\mathrm{N}$ deprivation on their ultrastructure. Numerous oil bodies, of various sizes, were present in both the N-deprived and control cells. However, their total volumes were substantially higher in the former (Figure 5). The oil bodies in the N-deprived cells were globular, compact, and mostly 0.1 to $0.3 \mu \mathrm{m}$ in diameter, but a few (2 to 5 per section) were much larger, up to $2.0 \mu \mathrm{m}$. In addition to causing significant changes in oil body contents, $\mathrm{N}$ deprivation strongly affected chloroplast ultrastructure. Diatoms are protists that contain typical secondary plastids surrounded by four membranes. Thus, they differ strongly from those of the algae sensu stricto (in particular, green algae such as Chlamydomonas) and vascular plants such as Arabidopsis, which contain simple plastids bounded by two membranes). In the control cells, although the cultures were in stationary phase, the chloroplast and thylakoid membranes were still highly organized. In contrast, the four enclosing membranes and thylakoid membrane system tended to be dispersed and poorly organized under $\mathrm{N}$ deprivation.

The main membrane lipids in plant chloroplasts are monogalactosyldiacylglycerol (MGDG) and digalactosyldiacylglycerol (DGDG). In Arabidopsis, these galactolipids are critical for the biogenesis of photosynthetic membranes, and provide sources of PUFA for the whole cell and phospholipid surrogates under phosphoruslimited conditions. Further, dramatic accumulations of DGDG accompanied by major reductions in membrane phospholipids have been observed in phosphorus-limited Arabidopsis, suggesting that the plant can substitute DGDG for phospholipids during phosphate starvation [38]. These galactolipids are synthesized by MGD and DGD galactosyltransferases/synthases, which also mediate membrane lipid remodeling [38]. Three genes encoding putative monogalactosyldiacylglycerol synthases (EC 2.4.1.46) were detected in the $P$. tricornutum genome: Phatrdraft_9619, Phatrdraft_54168 and Phatrdraft_14125, which showed 6-, 6and 9-fold decreases in transcript levels, respectively, under $\mathrm{N}$ deprivation. These results imply that membrane lipid remodeling, mediated by reductions in the expression of MGDG and/or DGDG galactosyltransferases, occurred in the $\mathrm{N}$-deprived cells. 

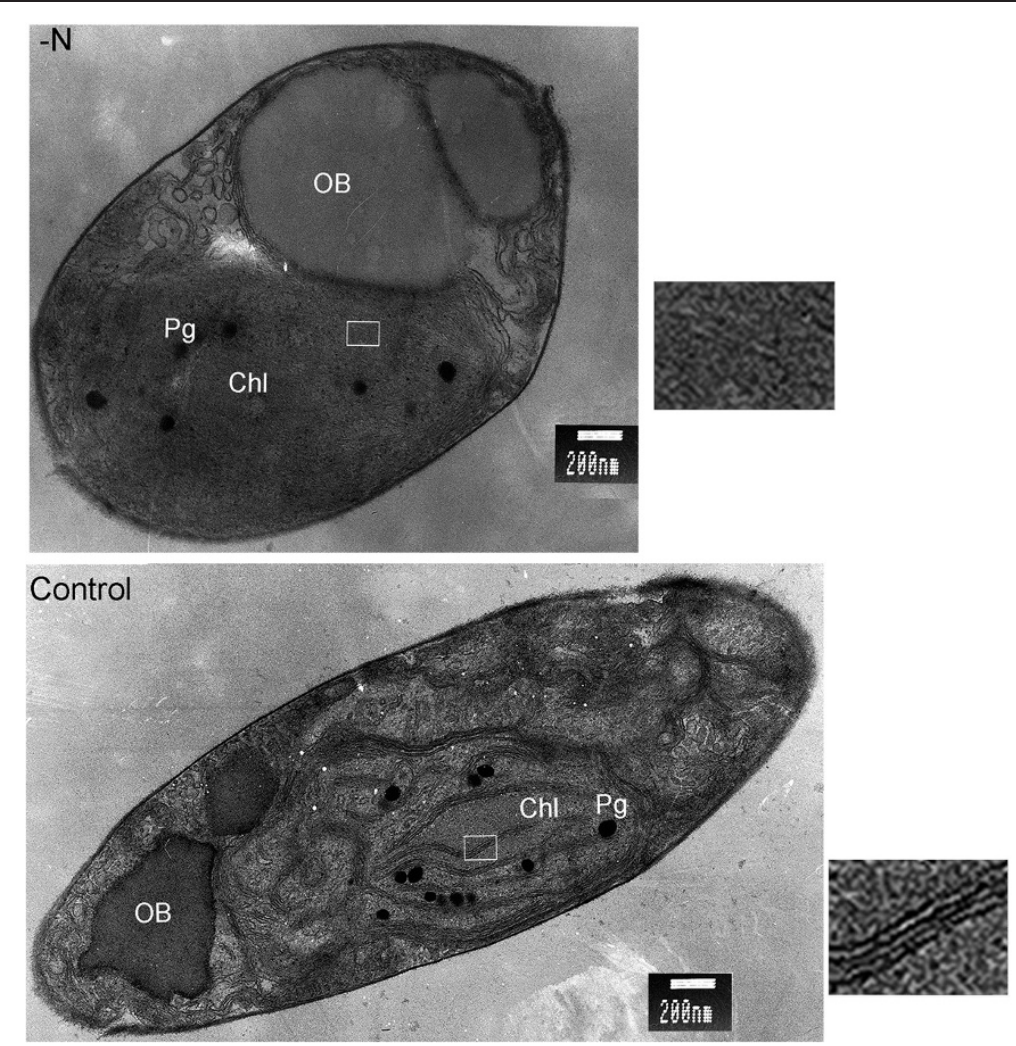

Figure $\mathbf{5}$ Effects of $\mathbf{N}$ deprivation on $\boldsymbol{P}$. tricornutum ultrastructure. Transmission electron micrographs showing subcellular structures of $\mathrm{N}$-deprived $(-\mathrm{N})$ and control P. tricornutum cells. OB, oil body; Chl, chloroplast; Pg, plastoglobule; boxed areas are magnified and showed to the right, demonstrating the dispersed thylakoid membranes under $-\mathrm{N}$. Bar $=200 \mathrm{~nm}$.

To further assess the contribution of membrane remodeling to lipid accumulation, total lipid extracts were separated into various lipid classes (Additional file 5: Figure S3) and analyzed by ESI/MS. The results showed that there were nine main classes of lipids in the $P$. tricornutum cells, and peak area measurements indicated that the proportions of MGDG and DGDG in the cells markedly decreased $50 \%$ under $\mathrm{N}$ deprivation, while the proportion of TAG (the major component of neutral lipids, mixed with photosynthetic pigments) appeared doubled.

We also analyzed the acquired transcription dataset to identify down-regulated genes that were annotated to the chloroplast. Out of 50 genes annotated to thylakoids, 45 were down-regulated, and only five were up-regulated. Our results demonstrate that lipid remodeling provides potential pathways for converting membrane lipids to substrates for TAG accumulation in $P$. tricornutum's responses to $\mathrm{N}$ deprivation.

Moreover, electron-dense osmiophilic globuli previously reported as plastoglobuli [39] were detected in the stroma of both $\mathrm{N}$-deprived and control cells, but there were fewer in the former (Figure 5). The functions of plastoglobuli and triggers for their formation are not well understood.
However, protein profiles of plastoglobuli indicate that metabolic enzymes accumulate in them and they have distinct lipoprotein structures [39]. In addition, overexpressing the plastoglobule structural protein fibrillin in plants results in increased frequencies of plastoglobuli [40], suggesting that fibrillin is involved in their formation. Transcripts encoding fibrillins in P. tricornutum (Phatrdraft 48066 and Phatrdraft_55153, encoding PAP-fibrillin-1 and PAPfibrillin-2) decreased 4- and 5-fold, respectively. This could at least partially explain the decrease of plastoglobuli in $\mathrm{N}$-deprived cells.

\section{Conclusion}

The overall goal of this study was to use genomic information and various analytical techniques to obtain an overview of the molecular mechanisms responsible for neutral lipid accumulation in the model diatom $P$. tricornutum induced by $\mathrm{N}$ deprivation. $\mathrm{N}$-deprived cultures of the diatom showed up to 2.4-fold increases in neutral lipid contents per cell, slightly higher cell density than controls and exhibited ultrastructural changes. In addition, the lipid composition of the N-deprived cells 
was more suitable for biodiesel production as they had higher proportions of saturated fatty acids.

Although the level of transcription is not directly proportional to the enzyme and the catalytic activity, recent study on another diatom T. pseudonana has shown a good correlation of proteomic and transcriptomic data [6]. In combination with biochemical investigations, our results could provide a global view of biosynthetic metabolic fluxes and unraveled significantly altered key metabolic pathways in the diatom following $\mathrm{N}$ deprivation. As summarized in Figure 6, it induced genes involved in carbon fixation, the TCA cycle and glycerolipid metabolism, but inhibited genes involved in light harvesting and photosynthesis. Energy resources necessary for carbon fixation and energy demand associated with the neutral lipid synthesis could be replenished from other sources such as elevated TCA cycle. A possible catabolism of carbohydrate reserves including chrysolaminarin may largely explain the neo-synthesis of TAG, in accordance with the up-regulation of glycolysis, coupled with the suppression of critical enzymes, mostly involved in lipid catabolism. The gene expression analyses, in combination with the ultrastructural and biochemical investigations, indicate that additional fatty acids could be produced by membrane remodeling. Thus, the findings provide indications of several mechanisms that contribute to the high lipid contents of microalgal cells under $\mathrm{N}$ deprivation.

The results also provide indications of strategies that could be used to enhance biodiesel production from microalgal cultures. The two-step culture method applied here dramatically increased neutral lipid accumulation, up to 2.4-fold, more than previous $\mathrm{N}$ limitation or continuous deficiency strategies. Furthermore, the inhibition of light harvesting under $\mathrm{N}$ deprivation indicates that high light intensity is not required for lipid accumulation, and the increase in carbon fixation suggests that increasing $\mathrm{CO}_{2}$ supplies during this stage should be advantageous.

In summary, results of this study show that diatom cultures with high levels of neutral lipids and increased cell proliferation rates can be obtained by nitrogen deprivation during the late exponential phase. They also provide indications of strategies that could be applied to manipulate the biosynthetic pathways of microalgae to generate cultures with high levels of lipids that may be suitable for biodiesel production.

\section{Materials and methods}

\section{Algal cultures}

Marine diatom Phaeodactylum tricornutum, with a fusiform morphotype, was obtained from the Freshwater Algal Culture Collection of the Institute of Hydrobiology, China (No. FACHB-863), and sub-cultured weekly, using a quarter of the preceding cultures as inocula, in Erlenmeyer

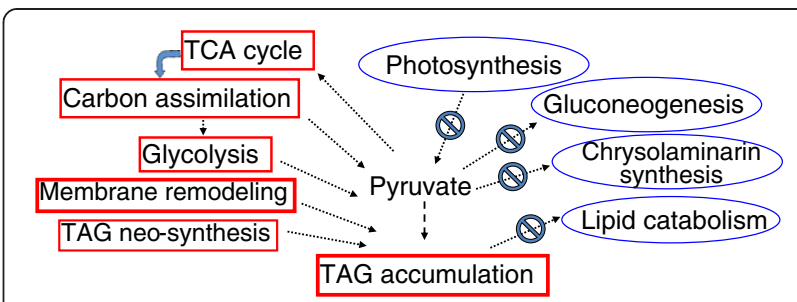

Figure 6 Schematic diagram indicating effects of $\mathrm{N}$ deprivation on processes involved in TAG accumulation based on transcriptional changes. Square and elliptical boxes indicate upregulation and down-regulation, respectively. " $\theta$ " indicates inhibition of the corresponding process.

flasks containing medium sterilized by passage through $0.22-\mu \mathrm{m}$ filters (Millipore). The growth medium (f/2-Si) contained $75 \mathrm{mg} \mathrm{NaNO}, 5.65 \mathrm{mg} \mathrm{NaH} \mathrm{NO}_{4} \cdot 2 \mathrm{H}_{2} \mathrm{O}, 4.16$ mg $\mathrm{Na}_{2}$ EDTA, $3.15 \mathrm{mg} \mathrm{FeCl} \cdot 6 \mathrm{H}_{2} \mathrm{O}, 0.01 \mathrm{mg} \mathrm{CuSO} \mathrm{Cl}_{4}$. $5 \mathrm{H}_{2} \mathrm{O}, 0.022 \mathrm{mg} \mathrm{ZnSO}_{4} \cdot 7 \mathrm{H}_{2} \mathrm{O}, 0.01 \mathrm{mg} \mathrm{CoCl}_{2} \cdot 6 \mathrm{H}_{2} \mathrm{O}$, $0.18 \mathrm{mg} \mathrm{MnCl}_{2} \cdot 4 \mathrm{H}_{2} \mathrm{O}, 0.006 \mathrm{mg} \mathrm{Na} \mathrm{MoO}_{4} \cdot 2 \mathrm{H}_{2} \mathrm{O}, 0.0005$ mg vitamin $B_{12}, 0.1 \mathrm{mg}$ vitamin $\mathrm{B}_{1}$ and $0.0005 \mathrm{mg}$ Biotin per liter in natural seawater, acquired from Daya Bay, Huizhou, China. The synchronized cultures were routinely cultivated in an artificial climate incubator at constant irradiance $\left(200 \mu \mathrm{mol}\right.$ photons $\left.\cdot \mathrm{m}^{-2} \cdot \mathrm{s}^{-1}\right)$ and temperature $\left(21 \pm 0.5^{\circ} \mathrm{C}\right)$ with $12 \mathrm{~h} / 12 \mathrm{~h}$ (1ight/dark) photoperiods.

For the experiments reported here, $1.5 \mathrm{~L}$ of algal culture in late-logarithmic growth phase was harvested 5 days after subculturing at 14:00 by centrifugation (4400 rpm for 10 min at $4^{\circ} \mathrm{C}$ ). The supernatant was discarded, the cell pellet was washed twice with $\mathrm{NaNO}_{3}$-free $\mathrm{f} / 2$-Si medium to completely remove the $\mathrm{N}$. Then the cells were collected by centrifugation $\left(4400 \mathrm{rpm}\right.$ for $15 \mathrm{~min}$ at $4^{\circ} \mathrm{C}$ ), and the resulting pellet was re-inoculated into $1.5 \mathrm{~L} \mathrm{~N}$-free $\mathrm{f} / 2$-Si medium. The resuspended culture was divided into two aliquots. One aliquot was split into six equal portions, which were transferred into six flasks, each containing $125 \mathrm{~mL} \mathrm{~N}$-free medium, and cultured under the above conditions (designated $\mathrm{N}$-deprived cultures). The other aliquot was also divided, transferred to six flasks and similarly treated, except that the medium was supplemented with $\mathrm{N}$, to provide controls. To maintain sampling consistency with respect to the diel cycle, cells were sampled at 14:00 every day.

\section{Cell density determination}

Cell numbers were counted using an Olympus microscope and Brightline Hemocytometer at the same time every day, in triplicates. Cell density $\left(\right.$ cell $\mathrm{mL}^{-1}$ ) was calculated as follows:

$$
\mathrm{CD}=(\mathrm{N} / 80) \times 400 \times 10^{4}
$$

where $\mathrm{CD}$ is cell density and $\mathrm{N}$ is the cell number per 80 grid cells. 
To facilitate counting, each sample was diluted 2-fold if the cell density exceeded $4 \times 10^{6}$ cells $\mathrm{mL}^{-1}$.

\section{Neutral lipid content analysis}

Nile red is a soluble phenoxazone lipid dye that partitions to cytoplasmic oil bodies in cells, and becomes fluorescent [41], the intensity of the fluorescence providing robust and convenient indications of the amount of neutral lipids present in various species, including the green microalga Chlorella $[14,41,42]$. In this study, the validity of the method for determining cellular lipid contents of P. tricornutum was first tested, by staining samples of the cultures with Nile Red (Sigma) following Chen $e$ al., with modifications [14]. Nile red $(30 \mu \mathrm{L}$ of a $0.1 \mathrm{mg} \mathrm{mL}^{-1}$ acetone solution) was added to $3 \mathrm{~mL}$ portions of cell cultures in triplicate, the resulting suspensions were mixed by rapid inversion, and incubated in darkness for $20 \mathrm{~min}$ at room temperature. The stained cell cultures were then transferred to cuvettes to determine their fluorescence intensity using a fluorescence spectrophotometer with $480 \mathrm{~nm}$ excitation wavelength. The optimal emission wavelength $(592 \mathrm{~nm})$ and staining time (20 min) were determined empirically, using nonstained cultures as auto-fluorescence controls. The relative fluorescence intensity values reported here reflect the differences in neutral lipid contents between stained and non-stained cells.

\section{Fatty acid composition analysis}

For fatty acid profiling analysis, total lipids were extracted from three independent biological replicates according to the method of Lepage and Roy [43], with modifications. Portions $(250 \mathrm{~mL})$ of the cultures were harvested after two days of $\mathrm{N}$ deprivation by centrifugation at $4400 \mathrm{rpm}$ at $4^{\circ} \mathrm{C}$ for $10 \mathrm{~min}$. Pellets were transferred to $10 \mathrm{~mL}$ tubes. Then $5 \mathrm{~mL} \mathrm{KOH}-\mathrm{CH}_{3} \mathrm{OH}$ solution was added to each tube, the cells were lysed by ultrasonication in an ice bath, the tubes were infused with nitrogen for $1 \mathrm{~min}$, tightly sealed, agitated and incubated at $75^{\circ} \mathrm{C}$ for $10 \mathrm{~min}$. After cooling to room temperature the upper phase was transferred to a $50 \mathrm{~mL}$ tube. The lower phase was subjected to two further washes with $5 \mathrm{~mL} \mathrm{KOH}-\mathrm{CH}_{3} \mathrm{OH}$, lysis and incubation, as described above, the resulting upper phases from each sample were pooled in a single tube, then $15 \mathrm{~mL}$ of $\mathrm{HCl}-\mathrm{CH}_{3} \mathrm{OH}$ solution was added, the solution was thoroughly mixed and incubated again at $75^{\circ} \mathrm{C}$ for $10 \mathrm{~min}$. N-hexane $(4 \mathrm{~mL})$ was added, the mixture was vortex-mixed, and allowed to settle. The upper phase was then transferred to a new $10 \mathrm{~mL}$ tube, while the lower phase was re-extracted with hexane. Then, the upper phases were pooled in a $10 \mathrm{~mL}$ tube, dried under a nitrogen stream in a Nitrogen Evaporator (Organomation, USA) and the residues were weighed and stored at $-80^{\circ} \mathrm{C}$ until analysis. Fatty acids were determined by gas chromatography-mass spectrometry (GC-MS) at the Institute of Microbiology, Guangdong, China. The chromatographic column used was a $30 \mathrm{~m} \times 0.25 \mathrm{~mm} \times 0.25 \mu \mathrm{m}$ DB-5 quartz capillary column. The column temperature

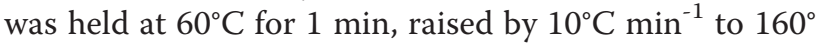
$\mathrm{C}$, and to a final temperature of $250^{\circ} \mathrm{C}$ at $2.5^{\circ} \mathrm{C} \mathrm{min}{ }^{-1}$. The injector temperature was $280^{\circ} \mathrm{C}$, and $1 \mu \mathrm{L}$ samples were injected, splitless. The mass spectrum transmission line temperature was $200^{\circ} \mathrm{C}$, and fatty acids were identified using the equipped NBS spectrum library, and quantitatively analyzed by determining integrated peak areas. Relative (percentage) contents of detected fatty acids were calculated by using the normalization method.

For lipid profiling analysis, total algal lipids were extracted following a protocol for Arabidopsis leaf tissue [44] with modifications. $200 \mathrm{ml}$ algal culture was harvested and the resulting pellets were quickly immersed in $3 \mathrm{ml}$ preheated $\left(75^{\circ} \mathrm{C}\right)$ isopropanol with $0.01 \%$ butylated hydroxytoluene (BHT) for $15 \mathrm{~min}$. The algal cells were then subjected to 80 rounds ( $5 \mathrm{~s}$ ) of ultrasonication in an Ultrasonic Crasher Noise Isolating Chamber, with $200 \mathrm{~W}$ output energy and $7 \mathrm{~s}$ intervals, during which the samples were placed in an ice bath to prevent lipid degradation. Then, $1.5 \mathrm{ml}$ chloroform and $0.6 \mathrm{ml}$ water were added to the cell detritus, and the suspensions were vortex-mixed and agitated in a shaking incubator at low temperature at $4^{\circ} \mathrm{C}$ for $40 \mathrm{~min}$. The mixtures were centrifuged at $4400 \mathrm{rpm}$ for $8 \mathrm{~min}$ to separate phases, and the lower phase was transferred to a new $10 \mathrm{ml}$ centrifuge tube. The remaining debris was extracted twice with $3 \mathrm{ml}$ chloroform/methanol (2:1) with $0.01 \%$ BHT, after which it was white. All extracts from samples representing cultures subjected to the same treatment were pooled, $1 \mathrm{ml}$ of $1 \mathrm{M} \mathrm{KCl}$ was added, the resulting mixture was shaken and centrifuged, and the upper phase was discarded. Then $2 \mathrm{ml}$ water was added to wash the extract, the mixture was centrifuged (12000 rpm for $10 \mathrm{~min}$ ) and the upper phase was removed. Finally, the organic solvents were evaporated by nitrogen and the residues were stored in a $-80^{\circ}$ $\mathrm{C}$ refrigerator until analysis. Total lipid extracts were separated into lipid classes according to Demandre et al. with modifications [45]. The dried algal lipid extracts were re-dissolved in $1 \mathrm{~mL}$ buffer A (isopropanol-hexane, 4:3), and the solutions were centrifuged at $14000 \mathrm{rpm}$ for $10 \mathrm{~min}$ to remove solid impurities, then transferred to a glass vial. Lipid classes were subsequently separated by HPLC using a silica column and a mobile phase consisting of a 20 -min linear gradient from $100 \%$ solution A to $100 \%$ B (isopropanol-hexane-water, 8:6:1.5), followed by isocratic solution B for a further $25 \mathrm{~min}$, with the column and sample collection system set at $20^{\circ} \mathrm{C}$. The total running time, including a 5 min re-equilibration 
step, was about $50 \mathrm{~min}$. The flow rate was $1.0 \mathrm{~mL} \mathrm{~min}^{-1}$ and $10 \mu \mathrm{L}$ samples were injected. Eluting fractions of lipids were detected spectrophotometrically at $205 \mathrm{~nm}$, manually collected in separate glass tubes, then stored at $-20^{\circ} \mathrm{C}$. Compounds in the lipid classes fractionated were diluted in isopropanol and identified using a Micromass Q-TOF micro system (Waters Co., UK) equipped with a syringe pump, LockSpray and ESI interface, operated in positive ionization mode. The mass spectrometer conditions were as follows: electrospray capillary voltage, $3.2 \mathrm{kV}$; ion source temperature, $110^{\circ} \mathrm{C}$; desolvation temperature, $350^{\circ} \mathrm{C}$; cone voltage, $15 \mathrm{~V}$; cone and collision gases, nitrogen and argon, at $60 \mathrm{~L} \mathrm{~h}^{-1}$ and $600 \mathrm{~L} \mathrm{~h}^{-1}$ flow rates, respectively. Sodium formate solution was used to calibrate the TOF mass spectrometer in positive electrospray ionization (ESI+) mode, and leucine enkephalin (L-EK) was used as an external mass calibration standard. Masses were scanned from 100 to $1100 \mathrm{amu}$ at approximately $0.4 \mathrm{scan} \mathrm{s}^{-1}$, and data were collected from m/z 90 to 1000 in continuum mode, and analyzed by Micromass MassLynx 4.1 software (Waters Co., USA). Peaks were also qualitatively analyzed by comparing determined molecular weights with related data in the LIPID MAPS Structure Database (LMSD).

\section{Observation of oil bodies}

To visualize oil bodies and assess their morphology, localization and numbers in the cultured cells Nile Red staining was applied $\left(0.1 \mathrm{mg} \mathrm{mL}^{-1}\right.$ in acetone in a 1:100 ratio) in dark conditions, using a similar procedure to that described for determination of the cells' neutral lipid contents. The mixtures were thoroughly mixed, applied to a glass slide, covered with a coverslip after about $10 \mathrm{~min}$, then observed under an LSM 510 META laser-scanning confocal microscope (Zeiss), with 543 $\mathrm{nm}$ excitation wavelength and 570-610 $\mathrm{nm}$ emission wavelength. Pictures were acquired randomly from at least 20 cells per sample, and typical images are presented here.

\section{RNA-Seq analysis}

After 48 hours of $\mathrm{N}$ deprivation, total RNA was extracted from six replicates of control and $\mathrm{N}$-deprived cultures using an RNeasy Plant Mini kit (QIAgen) following the manufacturer's instructions. The RNA samples were treated with QIAgen RNase-free DNase I during extraction, and the extracted mRNA was enriched using oligo (dT) magnetic beads. After adding fragmentation buffer, the mRNA was fragmented into short sequences (about $200 \mathrm{bp}$ ), first-strand cDNA was synthesized using random hexamer-primers and the mRNA fragments as templates, and then second strands were synthesized. The resulting double-stranded cDNA was purified with a QIAquick PCR extraction kit and subjected to end repair and single nucleotide A (adenine) addition. Finally, sequencing adaptors were ligated to the fragments, the required fragments were purified by agarose gel electrophoresis, enriched by PCR amplification, and sequenced using a HiSeq ${ }^{\mathrm{TM}} 2000$ (Illumina) instrument, with default quality parameters, at the BGI (Shenzheng), China.

\section{Validation of RNA-Seq data by quantitative real-time PCR} Triplicate portions $(2 \mu \mathrm{g})$ of total RNA were reversetranscribed using random hexamer primers and an Omniscript reverse transcription kit (QIAgen). Target genes (and $\beta$-actin as a housekeeping marker) were then subjected to quantitative PCR amplification in 96-well optical reaction plates in $20 \mu \mathrm{L}$ mixtures per well, using a SYBR Green Kit (Takara Bio) following the manufacturer's instructions and a 7300 Sequence Detection System (Applied Biosystems/Life Technologies). The threshold cycle $(\mathrm{Ct})$ for each well was measured, and the mRNA levels of the target genes in N-deprived cells, relative to those of controls, were quantified after normalization to $\beta$-actin.

RNA-Seq data processing and metabolic pathway analysis The draft $P$. tricornutum reference genome was obtained from http://genome.jgi-psf.org/Phatr2/Phatr2.download.ftp. html. The sequence is composed of "finished chromosomes" (Phatr2) and "unmapped sequences" (Phatr2_bd), which were annotated separately, and both were included in this study for comprehensive analysis. We aligned RNA short reads (11751810 in total) with the reference genome using SOAP 2.21 and default parameters. All original and mapped data are available at http://guanlab.ccmb. med.umich.edu/data/Yang_N_deprivation/. This resulted in 10786429 mapped reads (91.79\%). Any gene with one or no reads was discarded as non-confident, and with this constraint we aligned 12312844 reads obtained from samples of N-deprived cultures, with 11326275 (91.99\%) mapped to the genome. RPKM (Reads Per Kilobase per Million mapped reads) values were estimated and $\log _{2}$-transformed [46] for each gene defined in the Phatr2 database. This allowed us to estimate the fold-change in their transcript levels under nitrogen deprivation. We downloaded the KEGG pathway database (a unique set of genes that can be confidently associated with each protein in the KEGG pathway repository) released on Jun 30, 2011. We compiled all KEGG-annotated genes for P. tricornutum, and all those of other species meeting an e-value cutoff constraint of 1e-5 identified by BLAST analysis, retaining the best hits. We then hand-curated these two sets to remove inconsistencies and incorrect annotations, which resulted in 3648 genes with a KEGG id in total, and transformed the expression fold-change to a color gradient, where red represents up-regulation and green down-regulation. The subcellular localization of the enzymes were based on the prediction in 
the GenBank database, the prediction softwares online including WoLF PSORT (http://wolfpsort.org/) and SignalP (http://www.cbs.dtu.dk/services/SignalP/), besides relevant references.

\section{Measurement of photosynthesis activity}

Chlorophyll fluorescence parameters sensitively reflect the instantaneous photosynthetic state of diatoms and their acclimation to current environmental conditions. Fv/Fm (the variable/maximum fluorescence ratio) indicates the maximum photochemical quantum yield of PSII reaction centers, reflecting the photosynthetic light energy conversion efficiency. Thus, it is a widely used index of photosynthetic performance and acclimation status [21]. Fo is the minimum fluorescence yield when PSII reaction centers are fully open. Damage to or irreversible loss of activity of PSII reaction centers will cause a decrease in the Fo value. Fm is the maximum fluorescence yield when PSII reaction centers are completely closed, thus it reflects the PSII electron transport capacity. $\mathrm{Fv}$ is the variable fluorescence $(\mathrm{Fv}=\mathrm{Fm}-\mathrm{Fo})$, reflecting reduction of the PSII primary electron acceptor QA, thus indicating the photochemical activity of PSII reaction centers. To measure these parameters, $P$. tricornutum cultures were kept in the dark for $20 \mathrm{~min}$, then exposed to a saturating light pulse $\left(3000 \mathrm{~mol} \cdot \mathrm{m}^{-2} \cdot \mathrm{s}^{-1}\right)$ for $1 \mathrm{sec}$ while the chlorophyll fluorescence intensities were measured with a Handy-PEA chlorophyll fluorimeter (Hansatech Instruments Ltd) following the manufacturer's recommendations.

\section{Ultrastructural analysis by transmission electron microscopy} Samples were fixed in $2 \% \mathrm{v} / \mathrm{v}$ glutaraldehyde, $2 \% \mathrm{w} / \mathrm{v}$ paraformaldehyde in $100 \mathrm{mM}$ sodium cacodylate buffer- $\mathrm{NaOH}, \mathrm{pH} 7.4$, for $2.5 \mathrm{~h}$ at $4^{\circ} \mathrm{C}$ and rinsed three times (20 min per rinse) with $130 \mathrm{mM}$ sucrose, $10 \mathrm{mM}$ 2-mercaptoethanol in $100 \mathrm{mM}$ sodium cacodylate buffer- $\mathrm{NaOH}, \mathrm{pH}$ 7.4. They were then post-fixed with $1 \%(\mathrm{w} / \mathrm{v})$ osmium tetroxide in $100 \mathrm{mM}$ sodium cacodylate buffer- $\mathrm{NaOH}, \mathrm{pH} 7.4$, rinsed three times (5 min per rinse) with ultrapure (Milli-Q) water and dehydrated through a graded series of acetone (20\%, 50\%, 70\%, $90 \%, 100 \% \mathrm{v} / \mathrm{v})$. After infiltration through a graded acetone/Epon/Spurr's epoxy resin series, samples were embedded in $100 \% \mathrm{w} / \mathrm{v}$ Spurr's epoxy resin and polymerized at $60^{\circ} \mathrm{C}$ for $24 \mathrm{~h}$. Ultrathin sections were prepared using a Diatome diamond knife on an 8800 Ultratome III (LKB Instruments) and stained with uranyl acetate and lead citrate. The stained sections (about 20 per treatment) were examined under a JEM-1200EX transmission electron microscope (JEOL), and images were recorded on 4489 film (Eastman-Kodak).

\section{Additional files}

\section{Additional file 1: DataSet of gene expression of $P$. tricornutum following $\mathrm{N}$ deprivation.}

Additional file 2: Figure S1. Distribution of the number of reads across different mRNA sequence size. Each box plot depicts the numbers of reads for each gene (log base 10) in an mRNA sequence size bin $(0-5,000 \mathrm{bp}$, bin size of $500 \mathrm{bp})$. The line shows the range from minimum to maximum. The box and bar shows the quantiles. All mRNAs of 5,000 or more nucleotides are classified as 5,000 and above. A) nitrogen deprived condition. B) normal condition.

Additional file 3: Table S1. Fold changes in the expression of some genes encoding enzymes involved in various metabolisms following $\mathrm{N}$ deprivation.

Additional file 4: Figure S2. Expression alteration in some metabolic pathways. A) nitrogen fixation pathway; B) carbon fixation pathway; C) TCA cycle. Genes were mapped to KEGG pathways through annotation records in KEGG as well as homology map. Numbers in the boxes represent the EC number of the gene. Red indicates increase in expression level and blue indicates decrease. The intensity of the color bar is linearly correlated to the expression level change ( $\log _{2}$ fold).

Additional file 5: Figure S3. Separation and identification of lipid classes. Chromatograms showing (A) Total lipids of control cells, (B) Total lipids of $\mathrm{N}$-deprived cells. Peak identifications are described in the text.

\section{Abbreviations}

RNA-seq: RNA-sequencing; MGDG: Monogalactosyldiacylglycerol; TCA: Tricarboxylic cycle; TAG: Triacylglycerol; PUFA: Polyunsaturated fatty acid; SFA: Saturated fatty acid; KEGG: Kyoto encyclopedia of genes and genomes; LHC: Light harvesting complex; PSII: Photosystem II; ROS: Reactive oxygen species; PEPC: Phosphoenolpyruvate carboxylase; OAA: Oxaloacetate; CCM: Carbon concentrating mechanism; GS: Glutamine synthetase; PEP: Phosphoenolpyruvate; ME: Malic enzyme; ACCase: Adetyl-CoA carboxylase; FAS: Fatty acid synthase; FTIR: Fourier transform infrared spectrometer; FBP: Fructose-1,6-bisphosphatase; DGAT: Diacylglycerol acyltransferase; PDAT: Phospholipid:diacylglycerol acyltransferase; PC: Phosphatidylcholine; RPKM: Reads per kilobase per million mapped reads; FA: Fatty acid; DW: Dry weight.

Competing interests

The authors declare that there are no competing interests.

\section{Authors' contributions}

$H L$ and $Y G$ designed the research; $Z Y, Y N, Y M, J X, M Z, W Y, J L, Y G$ and $H L$ performed research; $Y G, Z Y$ and $H L$ contributed analytic and computational tools; $Z Y, Y N, W Y, J L, S L, Y G$ and $H L$ analyzed data; ZY, YG and HL wrote the paper. All authors read and approved the final manuscript.

\section{Acknowledgements}

This work was supported by the National Technologies Program (2011BAD14B03), Science and Technology Project of Guangdong (2010B030600005, 2009B020301002, 2009B050600005), and the Fundamental Research Funds for the Central Universities. Y.G. is supported by NIH 1R21NS082212-01 and EU-FP VII Systems Biology of Rare Disease.

Received: 25 September 2012 Accepted: 1 May 2013

Published: 4 May 2013

\section{References}

1. Falkowski PG, Barber RT, Smetacek V: Biogeochemical controls and feedbacks on ocean primary production. Science 1998, 281:200-206.

2. Lebeau T, Robert JM: Diatom cultivation and biotechnologically relevant products. Part I: cultivation at various scales. Appl Microbiol Biotechnol 2003, 60:612-623.

3. Chisti Y: Biodiesel from microalgae. Biotechnol Adv 2007, 25:294-306.

4. Bowler C, Allen AE, Badger JH, Grimwood J, Jabbari K, Kuo A, Maheswari U, Martens C, Maumus F, Otillar RP, et al: The Phaeodactylum genome reveals the evolutionary history of diatom genomes. Nature 2008, 456:239-244. 
5. Yu ET, Zendejas FJ, Lane PD, Gaucher S, Simmons BA, Lane TW: Triacylglycerol accumulation and profiling in the model diatoms Thalassiosira pseudonana and Phaeodactylum tricornutum (Baccilariophyceae) during starvation. J Appl Phycol 2009, 21:669-681.

6. Hockin NL, Mock T, Mulholland F, Kopriva S, Malin G: The response of diatom central carbon metabolism to nitrogen starvation is different from that of green algae and higher plants. Plant Physiol 2012, 158:299-312.

7. Valenzuela J, Mazurie A, Carlson RP, Gerlach R, Cooksey KE, Peyton BM, Fields MW: Potential role of multiple carbon fixation pathways during lipid accumulation in Phaeodactylum tricornutum. Biotechnol Biofuels 2012, 40:5.

8. Mock T, Kroon BMA: Photosynthetic energy conversion under extreme conditions-I: important role of lipids as structural modulators and energy sink under $\mathrm{N}$-limited growth in Antarctic sea ice diatoms. Pytochemistry 2002, 61:41-51.

9. Qian K, Michael AB: Light and nitrogen deficiency effects on the growth and composition of Phaeodactylum tricornutum. Appl Biochem Biotechnol 1993, 38:93-103.

10. Larson TR, Rees TAV: Changes in cell composition and lipid metabolism mediated by sodium and nitrogen availability in the marine diatom Phaeodactylum tricornutum (Bacillariophyceae). J Phycol 1996, 32:388-393.

11. James GO, Hocart CH, Hillier W, Chen H, Kordbacheh F, Price GD, Djordjevic MA: Fatty acid profiling of Chlamydomonas reinhardtii under nitrogen deprivation. Bioresour Technol 2011, 102:3343-3351.

12. Miller R, Wu G, Deshpande RR, Vieler A, Gartner K, Li X, Moellering ER, Zauner S, Cornish AJ, Liu B, et al: Changes in transcript abundance in Chlamydomonas reinhardtii following nitrogen deprivation predict diversion of metabolism. Plant Physiol 2010, 154:1737-1752.

13. De Martino A, Meichenin A, Shi J, Pan K, Bowler C: Genetic and phenotypic characterization of Phaeodactylum tricornutum (Bacillariophyceae) accessions. J Phycol 2007, 43:992-1009.

14. Chen W, Zhang C, Song L, Sommerfeld M, Hu Q: A high throughput Nile red method for quantitative measurement of neutral lipids in microalgae. J Microbiol Meth 2009, 77:41-47

15. Siron R, Giusti G, Berland B: Changes in the fatty acid composition of Phaeodactylum tricornutum and Dunaliella tertiolecta during growth and under phosphorus deficiency. Mar Ecol Prog Ser 1989, 55:95-100.

16. Grima EM, Medina AR, Giménez AG, González MJl: Gram-scale purification of eicosapentaenoic acid (EPA,20,5n-3) from wet Phaeodactylum tricornutum UTEX 640 biomass. J Appl Phycol 1996, 359:367.

17. Peltier G, Schmidt G: Chlororespiration: an adaptation to nitrogen deficiency in Chlamydomonas reinhardtii. Proc Natl Acad Sci U S A 1991, 88:4791-4795

18. Roberts K, Granum E, Leegood R, Raven J: Carbon acquisition by diatoms. Photosyn Res 2007, 93:79-88.

19. Tretter L, Adam-Vizi V: Alpha-ketoglutarate dehydrogenase: a target and generator of oxidative stress. Philos Trans R Soc Lond B Biol Sci 2005, 360:2335-2345.

20. Niyogi KK: Photoprotection revisited: genetic and molecular approaches. Ann Rev Plant Physiol Plant Mol Biol 1999, 50:333-359.

21. Campbell D, Hurry V, Clarke A, Gustafsson P, Oquist G: Chlorophyl fluorescence analysis of Cyanobacterial photosynthesis and acclimation. Microbiol Mol Biol Rev 1998, 62:667-683.

22. Grossman A, Takahashi H: Macronutrient utilization by photosynthetic eukaryotes and the fabric of interactions. Ann Rev Plant Physiol Plant Mol Biol 2001, 52:163-210.

23. Rivoal J, Turpin DH, Plaxton WC: In vitro phosphorylation of phosphoenolpyruvate carboxylase from the green alga Selenastrum minutum. Plant Cell Physiol 2002, 43:785-792

24. Reinfelder JR, Milligan AJ, Morel FMM: The role of the C4 pathway in carbon accumulation and fixation in a marine diatom. Plant Physio/ 2004 135:2106-2111.

25. McGinn PJ, Morel FMM: Expression and inhibition of the carboxylating and decarboxylating enzymes in the photosynthetic C4 pathway of marine diatoms. Plant Physiol 2007, 146:300-309.

26. Hopkinson B, Dupont C, Allen A, Morel F: Efficiency of the CO2 concentrating mechanism of diatoms. Proc Natl Acad Sci U S A 2011, 108:3830-3837.

27. Kroth PG, Chiovitti A, Gruber A, Martin-Jezequel V, Mock T, Parker MS, Stanley MS, Kaplan A, Caron L, Weber T, et al: A Model for carbohydrate metabolism in the diatom Phaeodactylum tricornutum deduced from comparative whole genome analysis. PLoS One 2008, 3:e1426.

28. Wynn J, Hamidt A, Ratledge C: The role of malic enzyme in the regulation of lipid accumulation in filamentous fungi. Microbiology 1999, 145:1911-1917.

29. Zhang Y, Adams IP, Ratledge C: Malic enzyme: the controlling activity for lipid production? Overexpression of malic enzyme in Mucor circinelloides leads to a 2.5 -fold increase in lipid accumulation. Microbiology 2007, 153:2013-2025.

30. Osteras M, Driscoll B, Finan T: Increased pyruvate orthophosphate dikinase activity results in an alternative gluconeogenic pathway in Rhizobium (Sinorhizobium) meliloti. Microbiology 1997, 143:1639-1648.

31. Jiang Y, Yoshida T, Quigg A: Photosynthetic performance, lipid production and biomass composition in response to nitrogen limitation in marine microalgae. Plant Physiol Biochem 2012, 54:70-77.

32. Minard K, McAlister-Henn L: Sources of NADPH in yeast vary with carbon source. J Biol Chem 2005, 280:39890-39896.

33. Li Y, Han D, Hu G, Dauvillee D, Sommerfeld M, Ball S, Hu Q: Chlamydomonas starchless mutant defective in ADP-glucose pyrophosphorylase hyper-accumulates triacylglycerol. Metab Eng 2010, 12:387-391.

34. Wang ZT, Ullrich N, Joo S, Waffenschmidt S, Goodenough U: Algal lipid bodies: stress induction, purification, and biochemical characterization in wild-type and starchless Chlamydomonas reinhardtii. Eukaryot Cell 2009, 8:1856-1868.

35. Guihéneuf F, Leu S, Zarka A, Khozin-Goldberg I, Khalilov I, Boussiba S: Cloning and molecular characterization of a novel acyl-CoA: diacylglycerol acyltransferase 1-like gene (PtDGAT1) from the diatom Phaeodactylum tricornutum. FEBS J 2011, 278:3651-3666.

36. Xu J, Francis T, Mietkiewska E, Giblin E, Barton D, Zhang Y, Zhang M, Taylor $D$ : Cloning and characterization of an acyl-CoA-dependent diacylglycerol acyltransferase 1 (DGAT1) gene from Tropaeolum majus, and a study of the functional motifs of the DGAT protein using site-directed mutagenesis to modify enzyme activity and oil content. Plant Biotechnol J 2008, 6:799-818.

37. Stahl U: Cloning and functional characterization of a phospholipid: diacylglycerol acyltransferase from Arabidopsis. Plant Physiol 2004 135:1324-1335

38. Kobayashi K, Awai K, Nakamura M, Nagatani A, Masuda T, Ohta H: Type-B monogalactosyldiacylglycerol synthases are involved in phosphate starvation-induced lipid remodeling, and are crucial for low-phosphate adaptation. Plant J 2009, 57:322-331.

39. Ytterberg AJ, Peltier JB, van Wijk KJ: Protein profiling of plastoglobules in chloroplasts and chromoplasts: A surprising site for differential accumulation of metabolic enzymes. Plant Physiol 2006, 140:984-997.

40. Rey P, Gillet B, Römer S, Eymery F, Massimino J, Peltier G, Kuntz M: Overexpression of a pepper plastid lipid-associated protein in tobacco leads to changes in plastid ultrastructure and plant development upon stress. Plant J 2000, 21:483-494

41. Greenspan P, Mayer EP, Fowler SD: Nile red: a selective fluorescent stain for intracellular lipid droplets. J Cell Biol 1985, 100:965-973.

42. Byrne SL, Foito A, Hedley PE, Morris JA, Stewart D, Barth S: Early response mechanisms of perennial ryegrass (Lolium perenne) to phosphorus deficiency. Ann Botany 2010, 107:243-254

43. Lepage $G$, Roy C: Improved recovery of fatty acid through direct transesterification without prior extraction or purification. J Lipid Res 1984, 25:1391-1396.

44. Li W, Wang R, Li M, Li L, Wang C, Welti R, Wang X: Differential degradation of extraplastidic and plastidic lipids during freezing and post-freezing recovery in Arabidopsis thaliana. J Biol Chem 2008, 283:461-468.

45. Demandre C, Tremolieres A, Justin A-M, Mazliak P: Analysis of molecular species of plant polar lipids by high-performance and gas liquid chromatography. Phytochemistry 1985, 24:481-485.

46. Babu MM: An Introduction to Microarray Data Analysis. In Computational Genomics: Theory and Application. Edited by Richard P. United Kingdom: Horizon Bioscience; 2004:225-249.

doi:10.1186/1754-6834-6-67

Cite this article as: Yang et al:: Molecular and cellular mechanisms of neutral lipid accumulation in diatom following nitrogen deprivation. Biotechnology for Biofuels 2013 6:67 